\title{
Guest Editor's Comments on Special Issue on Nonuniform Sampling
}

\author{
Farokh Marvasti \\ Advanced Communication Research Institute (ACRI) and EE Department \\ Sharif University of Technology, Tehran, Iran \\ http://acri.sharif.edu, marvasti@sharif.edu
}

It is a great pleasure for me to act as a guest editor for our journal on my favourite topic, Nonuniform Sampling. Although there are several excellent journal review papers on sampling theory $[3,9,19]$, there are no specific review papers on nonuniform sampling. However, there are several books and book chapters on this topic $[11,12,13,14]$. Recently, the concept of nonuniform sampling has found applications in compressed sensing [2], real field error correcting codes [16], impulsive noise cancellation [21, 22], sparse blind source separation [1], rate of innovation [20], and missing sampling problems for sparse signals [8] ${ }^{1}$. When Prof. Jerri, my dear friend, asked me to act as a guest editor for a special issue two years ago, I gladly accepted. The result of open call and personal soliciting are the following six papers on this issue.

The papers are organized in an increasing order of randomness. The first paper by Amini and Marvasti deals with periodic nonuniform sampling for multiband (including low-pass and band-pass) signals. In this paper we show that, depending on the positions on periodic nonuniform samples, the generated matrix may or may not be invertible. In any case, iterative methods converge to the pseudo-inverse. In case the matrix has a poor condition number, a finite number of iterations approximates the pseudo-inverse.

The second paper by Boumenir deals with nonuniform sampling points that can be associated with the eigen-values of a Sturm-Liouville boundary condition differential equation. He finds the condition of the sampling set that can be associated with the eigen-values and therefore perfect reconstruction can be guaranteed if these sampling sets are the nonuniform samples of a bandlimited signal of a specific bandwidth. He further finds conditions such that the sampling set can recover signals belonging to Paley-Wiener Space of any bandwidth.

The third paper by Thévenaz, Bierlaire, and Unser, although theoretical in nature, deals with a practical problem of matching two segments of an image (solving a puzzle) by maximizing the mutual information of samples of the two segments. This paper shows that a pseudo-random sequence called Halton sampling points (special case of nonuniform sampling) performs better than uniform

\footnotetext{
${ }^{1}$ Unfortunately, many of the previous and similar works are missing in these two papers, such as [18], papers by Gröchenig, Feichtinger, and Ferreira; see [12].
} 
or random samples. In this paper, Quadratic B-splines are used as kernels of the joint density.

The fourth paper by Lyubarskii and Madych is the generalization of the Poisson Sum Formula to nonuniform samples that form a Riesz basis. This work is a generalization of the works of $[4,7,10,15]$.

The fifth paper by Aldroubi and Leonetti is on analysis of jitter on nonuniform points that form a shift invariant space. They find bounds under which jitter does not alter the sampling set of the corrupted nonuniform samples. They also perform error analysis when the jitter is not known, and find conditions on the reconstruction kernel to achieve zero error when the jitter norm goes to zero.

The last paper by Holger Rauhut is on the new and emerging field of compressed sensing ${ }^{2}[5,6]$ where the observed samples of a sparse signal can be much lower than the apparent Nyquist rate. Holger assumes sparsity in the Discrete Fourier Domain and the observation set is random sampling. He derives a lower bound such that if the random sampling rate is lower than this bound, reconstruction schemes are not reliable.

\section{Happy Random Sampling!}

\section{References}

[1] M. Babaie-Zadeh and C. Jutten, A general approach for mutual information minimization and its application to blind source separation, Signal Processing, 85(5), 975-995, 2005.

[2] R. Baraniuk, A lecture on compressive sensing, IEEE Signal Process. Magazine, 24(4), 118-121, 2007.

[3] P. L. Butzer and R. L. Stens, Sampling theory for not necessarily bandlimited functions: A historical overview, SIAM Review, 34(1), 40-53, 1992.

[4] P. Butzer and A. Gessinger, The approximate sampling theorem, Poisson's sum formula, a decomposition theorem for Parseval's equation and their interconnections", Proc. of SAMPTA 95, Jurmala, Latvia, 1995.

[5] E. J. Candes, Compressive sampling, Proc. of the International Congress of Math., Madrid, Spain, 2006.

\footnotetext{
${ }^{2}$ According to Holger Rauhut: "The ideas of compressed sensing are not completely new. There are actually many precursors. For instance, the use of $l_{1}$ minimization seems to be first mentioned in the Ph.D. thesis of Logan in 1965. $l_{1}$ minimization was further used by geophysicists starting in 1970s. In statistics the field of variable selection has introduced $l_{1}$ minimization (called LASSO) and greedy algorithms (stepwise, forward regression, and projection pursuit regression, etc) in the 1980s and 1990s. The new contribution of compressed sensing consists in the type of applications, and in rigorous (and often fascinating) proofs of recovery results using new concepts and connections to other fields of mathematics." The paper by [17] is, in a sense, compressed sensing for nonlinear transformations.
} 
[6] D. Donoho, Compressed sensing, IEEE Trans. Inf. Theory, 52(4), 12891306, 2006.

[7] H. Feichtinger, Parseval's relationship for nonuniform samples of signals with several variables, IEEE Trans. Signal Process., 40(5), 1262-1263, 1992.

[8] O. G. Guleryuz, Nonlinear approximation based image recovery using adaptive sparse reconstructions and iterated denoising, Parts I and II, IEEE Trans. Image Process., 15(3), 539-571, 2006.

[9] A. Jerri, The Shannon sampling theorem: Its various extensions and applications: A tutorial review, Proc. of the IEEE, 65(11), 1565-1596, 1977.

[10] F. Marvasti, The extension of Poisson sum formulas to nonuniform samples, Proc. 5th Aachener Kolloquium, Aachen, Germany, 1984.

[11] F. Marvasti, A Unified Approach to Zero-Crossings and Nonuniform Sampling of Single and Multidimensional Signals and Systems, Nonuniform, Chicago, 1987.

[12] F. Marvasti, Nonuniform Sampling. In R. J. Marks II, Ed., Advanced Topics in Shannon Sampling and Interpolation Theory, Springer Verlag, New York, 1993.

[13] F. Marvasti, Fast Packet Network: Data, Image, and Voice Signal Recovery. In F. Fröhlich and A. Kent, Eds., Encyclopaedia of Telecommunications,7, Marcel Dekker, 453-479, 1994.

[14] F. Marvasti, Nonuniform Sampling: Theory and Practice, Kluwer/ Plenum, New York, 2001.

[15] F. Marvasti and L. Chuande, Parseval relationship of nonuniform samples of one- and two-dimensional signals, IEEE Trans. on Acoust., Speech, Signal Process., 38 (6) ,1061-1063, 1990.

[16] F. Marvasti, M. Hasan, M. Echhart and S. Talebi, Efficient algorithms for burst error recovery using FFT and other transform kernels, IEEE Trans. Signal Process., 47(4), 1065-1075, 1999.

[17] F. Marvasti and A. K. Jain, Zero crossings, bandwidth compression, and restoration of nonlinearly distorted band-limited signals, Journal of Opt. Soc. of America, 3(5), 651-654, 1986.

[18] F. Marvasti, C. Liu and G. Adams, Analysis and recovery of multidimensional signals from irregular samples using nonlinear and iterative techniques, Signal Processing, 36, 13-30, 1994. 
[19] M. Unser, Sampling- 50 years after Shannon, Proc. of the IEEE, 88(4), 569-587, 2000.

[20] M. Vetterli, P. Marziliano and T. Blu, Sampling signals with finite rate of innovation, IEEE Trans. Signal Process., 50(6), 1417-1428, 2002.

[21] S. Zahedpour, F. Marvasti and M. Babaie-Zadeh, A novel spike noise cancellation based on the iterative method, Proc. of SAMPTA 2007, Thessaloniki, Greece, 2007.

[22] S. Zahedpour, S. Feizi, A. Amini, M. Ferdosizadeh, and F. Marvasti, Impulsive noise cancellation based on soft decision and recursion, IEEE Trans. Instrumentaiton and Measurement, most likely to appear in 67(12), 2008. 